\title{
Multipoint weak signal acquisition based on orthogonal vector of the lock-in amplifier
}

\author{
Chao Sun ${ }^{1,2, a}$, You-Ping Chen ${ }^{1, b}$, Gang Zhang ${ }^{1, c, \uparrow}$, Feng Wang ${ }^{1, d}$, \\ Guang-Sen Liu ${ }^{1, \mathrm{e}}$ and Jian-Jun Ding ${ }^{2, \mathrm{f}}$ \\ ${ }^{1}$.School of Mechanical Science and Engineering, \\ Huazhong University of Science and Technology, Wuhan 430074, China \\ 2.School of Physics and Information Engineering, \\ Jianghan University, Wuhan 430056, China \\ aD201377130@hust.edu.cn, ${ }^{b}$ ypchen@mail.hust.edu.cn, ${ }^{c}$ zhanggang@hust.edu.cn

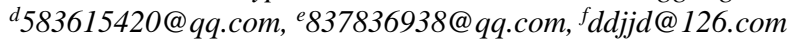 \\ ‘zhanggang@hust.edu.cn
}

\begin{abstract}
In the optical fiber sensor signals, most of them are weak. Generally, they are converted to weak current or voltage through the sensor unit. But the signal is too weak to measure because of the sensing unit background noise, circuit noise and external interference noise which is stronger than the useful signal. It is unable to detect the weak signal only by the amplification because amplification of the measured signal also amplified the noise. In this paper, a multi point acquisition system of weak signal is designed, which is based on the quadrature vector type lock-in amplifier, it can detect the weak signal effectively and effectively.
\end{abstract}

Keywords: weak signal, quadrature vector type, lock-in amplifier

\section{Introduction}

The weak signal mainly refers to the signal that is drowned by the noise, and it is relative to the noise. Weak Signal Detection (WSD) is a specially technology that designed suppression the noise signal. [1] The main task of weak signal detection is raising SNR (Noise Ratio Signal) which is the effective value of ratio of the effective signal $\mathrm{S}$ to the value of noise $\mathrm{N}$ that is $\mathrm{SNR}=\mathrm{S} / \mathrm{N}$.

Amplifier Lock-in (LIA) is a synchronous coherent detector designed by the principle of cross-correlation. [2] The lock-in amplifier consists of pre-amplifier, band pass filter, phase detector, low-pass filter, phase shift circuit and so on. Its structure is shown in Fig. 1. 


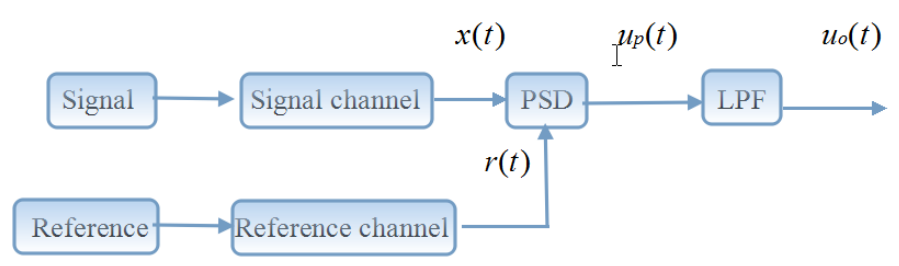

Fig 1 LIA structure

Quadrature lock-in amplifier need two phase sensitive detectors, which the input signal is the same, but difference phase with the 90 degrees, in the in-phase channel, the reference input phase is $\theta$ and quadrature channel phase is $\theta+90$ degrees. The amplitude and phase of the detected signal can be calculated by the two channels of QLIA.[3]

\section{System Design}

QLIA calculates the amplitude and phase of the signal by two orthogonal components, which can avoid the variable phase shift of the reference signal and avoid the effect of phase shift on the accuracy of the measurement. It can be seen from the formula, LIA output proportional to $\cos \theta$, so the measurement error of the $\theta$ will directly affect the measured signal amplitude measurement, it can be seen from equation, the QLIA measure the amplitude of the signal is not affected by the influence of measurement error in $\theta .[4][5]$

A signal in the form of time division multiplexing is put to 5 collection points. The duty cycle is $20 \%$ by means of the pulse. The signal that the collection comes back is first band-pass filter. Then the $\mathrm{Vp}-\mathrm{p} 100 \mathrm{~Hz}$ is $2 \mathrm{~V}$ and the sinusoidal signal of its lagging 90 degree signal is locked and amplified. LIA structure realization is shown in Fig 2. 


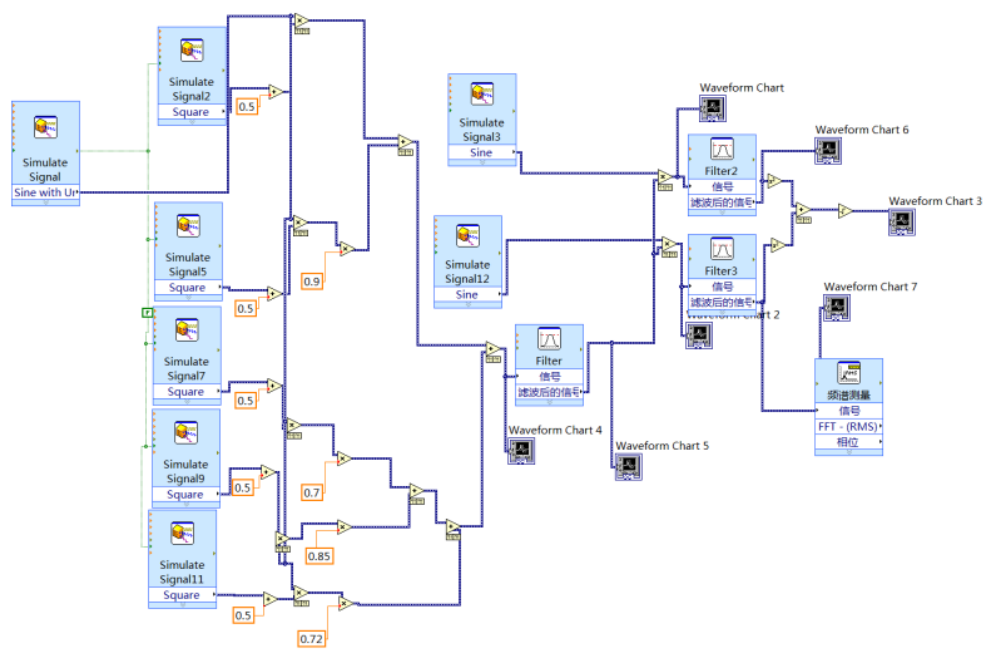

Fig 2 LIA structure realization

In this paper, a multi point acquisition system of weak signal is designed, which is based on the quadrature vector type lock-in amplifier, QLIA calculates the amplitude and phase of the signal by two orthogonal components, which can avoid the variable phase shift of the reference signal and avoid the effect of phase shift on the accuracy of the measurement. it can detect the weak signal efficiently and effectively. Modulation signal is shown in Fig 3. Methane gas absorption coefficient is shown in Fig 4.

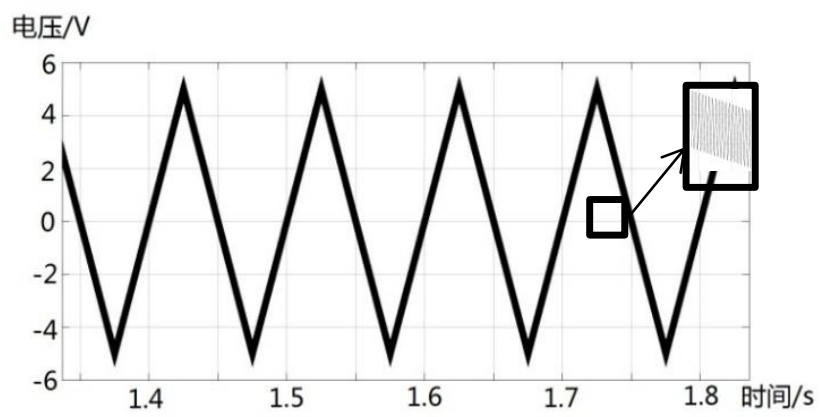

Fig 3 Modulation signal 


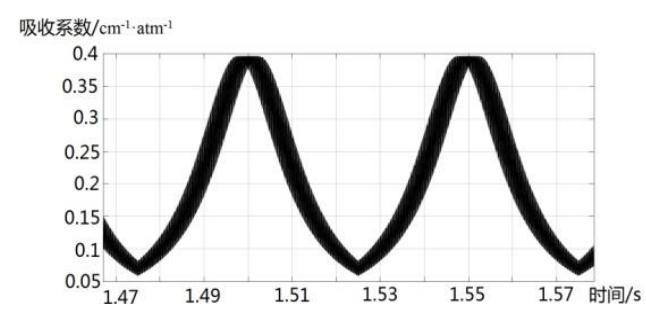

Fig 4 Methane gas absorption coefficient

\section{Simulation Result}

Fig5 show the light intensity changes with reference to the gas chamber and the detection chamber in Fig 5 and Fig 6 respectively, in which case the methane gas volume fraction is 0.1 .

With reference to the gas chamber, light intensity modulated signal changes at the same time, and the change cycle of the modulation signal and trends are the same. Detecting the light intensity in the gas chamber at half the height of the image there is a clear absorption phenomenon, and exhibit cyclical changes, the same period and the same modulation signal. Fig 6 is the result of the second harmonic of the image signal after the detection of the gas chamber, it can be seen the outline of the image is vague, since the introduction of the triangular wave components in the frequency.

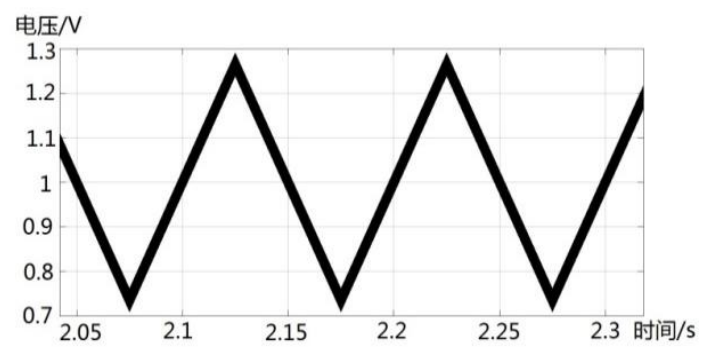

Fig. 5 Light intensity in reference gas chamber

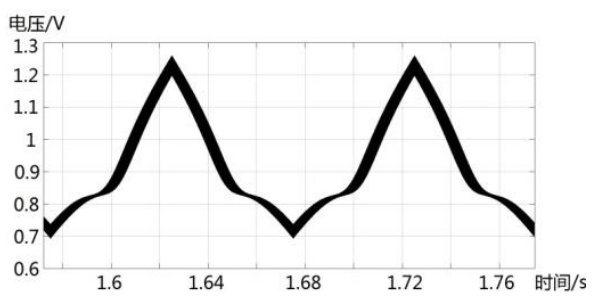

Fig. 6 Light intensity in detective gas chamber 
Fig 8 is obtained after the differential operational by detector signal and the reference gas chamber. Fig 9 shows the difference image after the second harmonic signal, and compare to Fig 7, the second harmonic signal after difference harmonic image is clearer by the triangular wave interference is smaller.

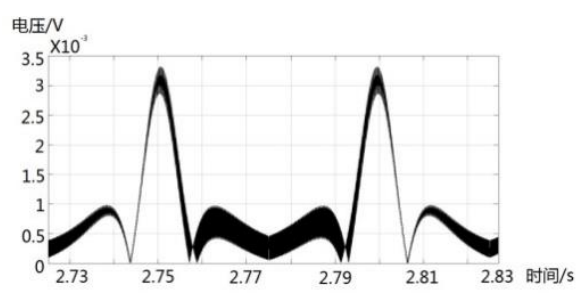

Fig 7 Second harmonic signal

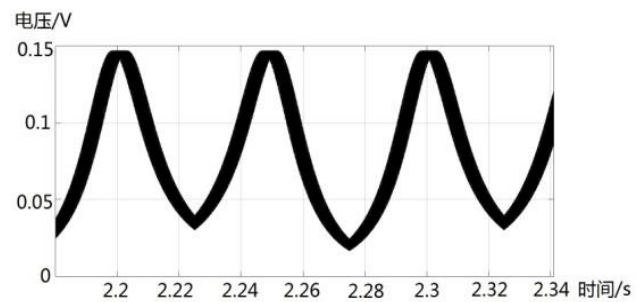

Fig 8 Differential operational signal

Fig 10 is obtained after processing of the second harmonic and LIA. It shows that the quadrature lock-in amplifier output stability is a constant stable signal, matching a previous derivation. When the methane gas volume fraction is set to 0.1 , the lock-in amplifier output voltage is $0.0498 \mathrm{~V}$, empathy can be obtained methane volume fraction of $0.05,0.01$ and 0.005 respectively lock-in amplifier output voltage is $0.0252 \mathrm{~V}, 0.00511 \mathrm{~V}$ and $0.00256 \mathrm{~V}$. The analysis of these points can be very high linearity, so the test program is reasonable.

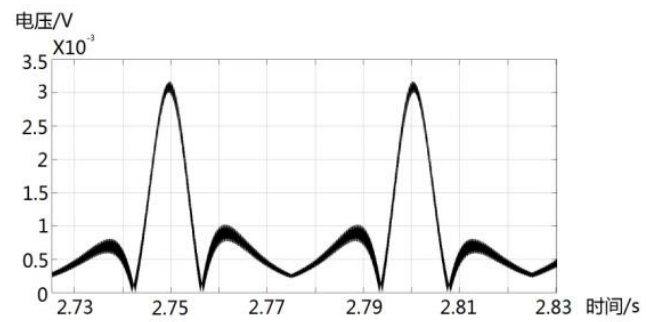

Fig 9 Second harmonic signal after differential 
Fig 10 LIA output

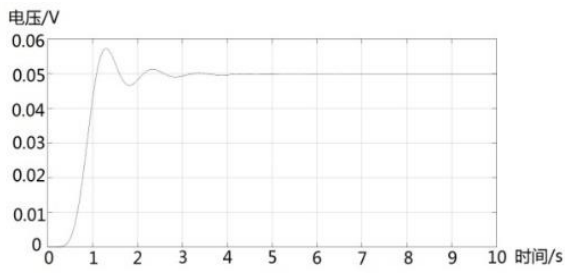

\section{Conclusion}

This work proposed a single dual-wavelength optical path difference optical structure. Since the light intensity at the detector through the normal voltage electrical conversion is less than a millivolt and signal intensity will be more weak after difference. The use of intensity modulation with second harmonic detection technology proposed is an effective weak signal processing method. Finally the Simulink simulation overall experimental program is carried out, and the results were studied reasonably come to the conclusion experimental program.

\section{References}

[1]. So S, Sani A A, Zhong L, et al. Laser spectroscopic trace-gas sensor networks for atmospheric monitoring applications[C]//ESSA Workshop. 2009, 9.

[2]. HAN X, YANG J. Methane gas sensor with spectrum absorption and signal processing method $[\mathrm{J}][\mathrm{J}]$. Journal of Xi'an University of Science and Technology, 2008, 3: 015.

[3]. Sa J. An optical fiber gas sensor based on harmonic detection[C]//Electric Information and Control Engineering (ICEICE), 2011 International Conference on. IEEE, 2011: 1169-1172.

[4]. Wei Y H H H J, Yanhe L Y L. Optical fiber gases sensing array with frequency modulated continuous wave technique $[\mathrm{J}][\mathrm{J}]$. Laser Journal, 2001, 2: 024.

[5]. Rahman M M, Khan S B, Jamal A, et al. Highly sensitive methanol chemical sensor based on undoped silver oxide nanoparticles prepared by a solution method[J]. Microchimica Acta, 2012, 178(1-2): 99-106. 